\title{
Do we need controlled clinical trials in pulmonary arterial hypertension?
}

\author{
N. Galiè
}

Uncontrolled studies on the effects of a treatment in a series of patients have a tendency to report favourable results, whereas case reports often inform about adverse effects. Accordingly, the recent publication of an open uncontrolled study reporting favourable effects of an inhaled prostacyclin analogue, iloprost, in patients with primary pulmonary hypertension (PPH) [1], did not come as a surprise. What came as a surprise was a methodologically comparable study on the same treatment, in a similar type of patient, in this issue of the European Respiratory Journal, with completely opposite conclusions [2]. How is such a discrepancy possible? The answer is straightforward, and holds in a single word: methodology.

Phase III controlled clinical trials (CCT) are required by both the scientific community and Regulatory Agencies as definitive proof of the safety and efficacy of new modalities of treatment. Uncontrolled, small phase II studies are performed at the very beginning of the development of new treatments to confirm the expected effects (proof of concept) and to assess the effective dose (dose finding). A well-designed phase III CCT incorporates elements such as prospective randomization, placebo-control and double-blindness, that ensure the reliability of the results. To increase the chance of success, a pivotal CCT needs an appropriate sample size based on the foreseeable changes of predefined primary end-points. Small, pilot phase III studies are usually required to gauge those changes and to test the appropriateness of the study design. A multicentre cooperation ensures a fast enrolment rate and reproducibility of results. The development of the current treatments of patients with pulmonary arterial hypertension (PAH), defined after the World Health Organization classification [3], have not followed all the golden rules of CCT. In fact, the favourable effect of oral anticoagulant therapy in patients with PPH or pulmonary hypertension associated with anorexigens, is based on the retrospective analysis of single centre studies [4-6]. These experiences showed an improvement of survival in the group treated with oral anticoagulant therapy, in comparison to a concurrent nonrandomized, untreated control group. The retrospective design has left some important unanswered questions, such as the optimal level of anticoagulation, that are currently left to the judgment of the physi-

Istituto de Cardiologia, Università di Bologna, Via Massarenti 9, 40138-Bologna, Italy. Fax: 3951344859. cian. On the other hand, the demonstration of the presence of thrombophylic predisposition [7-9] and of thrombotic changes in the microcirculation [10] and the elastic pulmonary arteries [11] of PPH patients, represent a strong rationale for the use of oral anticoagulant therapy in these subjects. In addition, the demonstration of the positive prognostic effect of calcium channel-blocking drugs (CCBs) in vasoreactive PPH patients (15-20\% of all PPH patients) has been shown in a single centre, nonrandomized, uncontrolled study [5]. In this case, the control group was represented by nonvasoreactive patients that had a worse spontaneous prognosis than vasoreactive patients [12]. Therefore, the favourable prognostic effect of $\mathrm{CCB}$ treatment shown in the study could have been exaggerated by an inappropriate comparison. The randomization of vasoreactive patients in treated and untreated groups would have given a more reliable demonstration of the positive effect of CCB. On the other hand, the demonstration of a consistent reduction in pulmonary arterial pressure by pharmacological test, as observed in vasoreactive patients, poses ethical questions about giving a placebo instead of $\mathrm{CCB}$ in these subjects. Unfortunately, the results of this study have occasionally been extended in clinical practice to nonvasoreactive patients or to patients not acutely tested. In fact, it is common experience that $\sim 50 \%$ of the PPH population is treated with $\mathrm{CCB}$, whereas only $15-20 \%$ of $\mathrm{PPH}$ patients are vasoreactive [13]. This may reduce the global beneficial impact of CCB treatment in $\mathrm{PPH}$ patients, due to the frequent and sometimes severe sideeffects of these drugs when administered in nonvasoreactive subjects [13].

The results of the first CCTs in PPH patients were published in 1990 by RUBIN et al. [14] and concerned the use of continuous intravenous infusion of epoprostenol (a prostacyclin analogue available for intravenous administration) by means of tunnelized catheters and portable pumps. In this multicentre study on 19 patients, the subjects randomized to the control group did not receive central venous catheter implantation and pumps for ethical reasons, hence the study was not blind. Nevertheless, the improvements in objective endpoints, like haemodynamics in the treated group compared to the untreated group, were considered reliable. BARST et al. [15] published a second multicentre CCT on the same form of treatment in 1996 involving 80 patients. Again, in this case, the control group did not undergo central venous catheter implantation so the study, although controlled, cannot be considered blind. 
Conversely, it showed an impressive prognostic advantage in the treated group (no deaths versus eight deaths in the untreated group) after 12 weeks, which makes a possible weakness of the design less relevant. Moreover, this second randomized study [15] reproduced the same favourable haemodynamic and functional effects (assessed by the six-minute walk test) of the first one [14], reinforcing the reliability of both studies.

The importance of these experiences went beyond their positive results because they demonstrated that it was possible to perform multicentre CCT in a relatively rare and severe disease like PPH. Moreover, they illustrated the reproducibility of results in studies with relatively small sample sizes. Recently, a third CCT on the effect of continuous intravenous infusion of epoprostenol in patients with PAH associated with scleroderma, has been published [16]. The improvements in haemodynamics and on the six-minute walk test were similar to those achieved in PPH. Beside these CCTs, uncontrolled experiences on epoprostenol treatment have been published in recent years both in PPH [17] and in PAH associated with scleroderma [18]. The results of these studies were in agreement with those of randomized studies, even if the length of the follow-up was much longer (years instead of months).

Uncontrolled phase III open studies should be considered as a confirmation and extension of observations made in CCTs and cannot be considered a reliable alternative. In fact, in these trials, the control group is constituted by historical or concurrent nonrandomized, untreated patients, or by baseline parameters of the treated patients. These forms of comparisons are not usually accepted by the scientific community or the Regulatory Agencies because they may suffer from selection bias, treatment bias and placebo effect. On the other hand, for ethical reasons randomized trials in a severe disease like PAH cannot last more than a few months and information on the long-term effect of treatments can only be collected in open-label studies. For these reasons, uncontrolled experiences should be considered as complementary, rather than alternative to CCT and ideally the open-label study should follow, rather than precede the randomized studies.

Currently, several CCTs are testing the effect of new forms of treatment in patients with PAH. These ongoing experiences are all multicentre, randomized, double-blind, and placebo-controlled. They are assessing the safety and efficacy of the following therapeutic modalities; subcutaneous prostacyclin $\left(\mathrm{PGI}_{2}\right)$ analogue (uniprost); inhaled $\mathrm{PGI}_{2}$ analogue (iloprost); oral $\mathrm{PGI}_{2}$ analogue (beraprost) and oral endothelin-1 receptor antagonist (bosentan). The designs of these studies are remarkably similar and they are split in a first randomized period (ranging from 3-4 months) and a second open label phase, in which all patients receive active treatment. This structure provides an opportunity to objectively assess the mid-term effects, as well as to establish safety and persistence of results in the long-term. The results of the randomized phase of the uniprost study have been presented at recent meetings of the European Society of Cardiology in Amsterdam, the European Respiratory Society in Florence and the American Heart Association in New Orleans. Four- hundred and seventy patients with PAH have been randomized in 40 centres worldwide and positive results have been shown in the treated group as assessed by symptom score, functional capacity and haemodynamics. Uniprost is infused subcutaneously by small portable pumps similar to those used for insulin by diabetic patients. The most frequent side-effects are pain and redness at the infusion site that can limit the increase in dose and prevent the use of the drug in $\sim 8 \%$ of patients. No effect on mortality was observed after 3 months; this could be explained by a relatively less sick population participating in this study compared to the early epoprostenol studies [15]. More insights on safety, tolerability and clinical effect of uniprost are expected from the ongoing open-label extension.

The results of a small controlled pilot phase III study on bosentan were presented at the American Heart Association meeting in New Orleans in November 2000; preliminary reports have shown that this oral compound exerts positive effects on functional capacity and haemodynamics in patients with PAH.

How should the results of uncontrolled studies be considered when they are published before the randomized studies, as in the case of inhaled iloprost [1, $19]$ and oral beraprost [20, 21] treatments? It is obvious that any attempt to find a better therapy in such a severe and debilitating disease as PAH should be welcomed. Nevertheless, the positive results of uncontrolled studies have to be considered as explorative and as an incentive to perform a randomized study, rather than as definitive acquisitions ready to be translated into everyday practice. One additional reason, besides methodological criticism, is that only positive experiences are usually published in peer reviewed journals, whereas negative experiences could not be reported. Instead, a multicentre CCT, if negative, could hardly go unrecognized by the experts.

In conclusion, in the history of treatments of pulmonary arterial hypertension, some "developmental violations" have characterized the experiences collected on oral anticoagulant therapy and calcium channelblockers that are currently considered "conventional therapy". Conversely, in the 1980s when those treatments were adopted, collaborative studies in a rare and severe disease like pulmonary arterial hypertension were quite difficult. Today, there is the opportunity to test new therapeutic modalities in a scientific way by controlled clinical trials, since multicentre studies have turned out to be feasible and reliable. Thus, only the new treatments that have shown positive results in controlled clinical trials should be introduced into clinical practice, whereas all the uncontrolled experiences should be considered exploratory.

\section{References}

1. Hoeper MM, Schwarze M, Ehlerding S, et al. Longterm treatment of primary pulmonary hypertension with aerosolized iloprost, a prostacyclin analogue. $N$ Engl J Med 2000; 342: 1866-1870.

2. Macherndl S, Kneussl M, Baumgartner H, et al. Longterm treatment of pulmonary hypertension with aerosolized iloprost. Eur Respir J 2001; 17: 8-13. 
3. Nomenclature Committee. Nomenclature and Classification of Pulmonary Hypertension. In: Rich S, ed. Primary Pulmonary Hypertension: Executive Summary from the World Symposium - 1998, 25-27. World Health Organization http://www.who.int/ncd/ cvd/pph.html

4. Fuster V, Steele PM, Edwards WD, Gersh BJ, McGoon MD, Frye RL. Primary pulmonary hypertension: natural history and the importance of thrombosis. Circulation 1984; 70: 580-587.

5. Rich S, Kaufmann E, Levy PS. The effect of high doses of calcium-channel blockers on survival in primary pulmonary hypertension. N Engl J Med 1992; 327: 7681.

6. Frank H, Mlczoch J, Huber K, Schuster E, Gurtner HP, Kneussl M. The effect of anticoagulant therapy in primary and anorectic drug-induced pulmonary hypertension. Chest 1997; 112: 714-721.

7. Eisenberg PR, Lucore C, Kaufman L, Sobel BE, Jaffe AS, Rich S. Fibrinopeptide A levels indicative of pulmonary vascular thrombosis in patients with primary pulmonary hypertension. Circulation 1990; 82: 841-847.

8. Christman BW, McPherson CD, Newman JH, et al. An imbalance between the excretion of thromboxane and prostacyclin metabolites in pulmonary hypertension. N Engl J Med 1992; 327: 70-75.

9. Friedman R, Mears JG, Barst RJ. Continuous infusion of prostacyclin normalizes plasma markers of endothelial cell injury and platelet aggregation in primary pulmonary hypertension. Circulation 1997; 96: 2782-2784.

10. Pietra GG, Edwards WD, Kay JM, et al. Histopathology of primary pulmonary hypertension. A qualitative and quantitative study of pulmonary blood vessels from 58 patients in the National Heart, Lung, and Blood Institute, Primary Pulmonary Hypertension Registry. Circulation 1989; 80: 1198-1206.

11. Moser KM, Fedullo PF, Finkbeiner WE, Golden J. Do patients with primary pulmonary hypertension develop extensive central thrombi? Circulation 1995; 91: 741-745.

12. Raffy $\mathrm{O}$, Azarian $\mathrm{R}$, Brenot $\mathrm{F}$, et al. Clinical significance of the pulmonary vasodilator response during short-term infusion of prostacyclin in primary pulmonary hypertension. Circulation 1996; 93: 484 488.

13. Galiè N, Ussia G, Passarelli P, Parlangeli R, Branzi A, Magnam B. Role of pharmacologic tests in the treatment of primary pulmonary hypertension. $\mathrm{Am}$ $J$ Cardiol 1995; 75: 55A-62A.

14. Rubin LJ, Mendoza J, Hood M, et al. Treatment of primary pulmonary hypertension with continuous intravenous prostacyclin (epoprostenol). Results of a randomized trial. Ann Intern Med 1990; 112: 485491.

15. Barst RJ, Rubin LJ, Long WA, et al. A comparison of continuous intravenous epoprostenol (prostacyclin) with conventional therapy for primary pulmonary hypertension. The Primary Pulmonary Hypertension Study Group. N Engl J Med 1996; 334: 296-302.

16. Badesch DB, Tapson VF, McGoon MD, et al. Continuous intravenous epoprostenol for pulmonary hypertension due to the scleroderma spectrum of disease. A randomized, controlled trial. Ann Intern Med 2000; 132: 425-434.

17. Shapiro SM, Oudiz RJ, Cao T, et al. Primary pulmonary hypertension: improved long-term effects and survival with continuous intravenous epoprostenol infusion. J Am Coll Cardiol 1997; 30: 343-349.

18. Klings ES, Hill NS, Ieong MH, Simms RW, Korn JH, Farber HW. Systemic sclerosis associated pulmonary hypertension: short- and long-term effects of epoprostenol (prostacyclin). Arthritis Rheum 1999; 42: 26382645.

19. Olschewski H, Ghofrani HA, Schmehl T, et al. Inhaled iloprost to treat severe pulmonary hypertension. An uncontrolled trial. German PPH Study Group. Ann Intern Med 2000; 132: 435-443.

20. Saji T, Ozawa Y, Ishikita T, Matsuura H, Matsuo N. Short-term hemodynamic effect of a new oral $\mathrm{PGI}_{2}$ analogue, beraprost, in primary and secondary pulmonary hypertension. Am J Cardiol 1996; 78: 244-247.

21. Nagaya N, Uematsu M, Okano Y, et al. Effect of orally active prostacyclin analogue on survival of outpatients with primary pulmonary hypertension. J Am Coll Cardiol 1999; 34: 1188-1192. 